\title{
A physical function intervention in community- dwelling disabled and older adults in a Medicaid waiver: results of a multi-site pragmatic hybrid type III cluster randomized trial comparing two implementation strategies
}

Sandra Spoelstra ( $\square$ spoelsts@gvsu.edu )

Grand Valley State University https://orcid.org/0000-0001-6850-5425

Monica Schueller

Grand Valley State University

Viktoria Basso

Grand Valley State University

Alla Sikorskii

Michigan State University

\section{Research Article}

Keywords: Adoption, Sustainability, Implementation, Implementation strategies, Physical function, Community-dwelling, Older adults, Medicaid waiver, cluster-randomized controlled trial

Posted Date: February 28th, 2022

DOI: https://doi.org/10.21203/rs.3.rs-1238935/v1

License: (c) (1) This work is licensed under a Creative Commons Attribution 4.0 International License. Read Full License 


\section{Abstract}

Background: Evidence-based interventions that optimize physical function for disabled and older adults living in the community who have difficulty with daily living tasks are available. However, to date, their implementation has been limited, particularly in state Medicaid home and community-based waiver programs. Furthermore, it is unknown whether implementation strategies commonly used in other settings would result in adoption and sustainability of these effective interventions in resourceconstrained Medicaid programs.

Methods: In a hybrid type III trial, waiver sites $(\mathrm{N}=18)$ were randomly assigned to implement the intervention using a bundle of strategies with either internal facilitation (IF) or IF and external facilitation (EF). Adoption and sustainability were assessed via stages of implementation completion (SIC) for each site. Clinician attitudes toward evidence-based practice and self-efficacy were evaluated among 539 registered nurses, social workers, and occupational therapists. Medicaid beneficiary outcomes of activities of daily living, depression, pain, falls, emergency department visits, and hospitalizations were evaluated in a sample of $\mathrm{N}=7,030$ as reflected by electronic health records data of the Medicaid waiver program. Linear mixed effects models were used to compare outcomes between trial arms while accounting for cluster-randomized design.

Results: The mean SIC scores were 72.22 (standard deviation [SD]=16.98) in the IF arm (9 sites), and $61.33(S D=19.29)$ in the IF+EF arm (9 sites). The difference was not statistically significant, but corresponded to the medium clinically important effect size Cohen's $d=0.60$. Clinician implementation outcomes of attitudes and self-efficacy did not differ according to the IF versus IF+EF. Beneficiary depression was reduced significantly in IF+EF arm compared to IF ( $p=.04,95 \%$ confidence interval for the difference $[0.01,0.24])$. No differences between trial arms were found for other beneficiary outcomes.

Conclusions: Level of facilitation did not enhance capacity for adoption and sustainability of an evidence-based intervention in an under-resourced Medicaid setting that cares for disabled and older adults. Improved beneficiary depression favored external and internal facilitation strategy, but no differences for other outcomes compared to interval facilitation only suggest that the addition of external facilitation may not be warranted.

Trial registration: ClinicalTrials.gov, NCT03634033; date registered August 16, 2018. https://clinicaltrials.gov/ct2/show/NCT03634033

\section{Contributions To The Literature}

- The results inform the use of implementation strategies for an evidence-based intervention that improves physical function and the ability to perform daily tasks, and assists disabled and older adults to remain living in the community.

- A long-term impact will be to enhance the waiver's capacity for practice innovations through development and testing of a bundle of implementation strategies to enhance adoption and 
sustainability of evidence-based interventions.

- The methodologic approach of measurement utilizing stages of implementation completion, comprised of three dimensions: quality, quantity, and timing, may be appropriate for a wide range of implementation and translational studies.

\section{Background}

The aging population in the United State is growing rapidly, [1] and nearly half report problems with physical function, which can lead to difficulty with daily tasks and often nursing home placement. [2-5] Evidence-based interventions that optimize physical function in settings that care for disabled and older adults are available yet rarely implemented, particularly in under-resourced settings.

One such evidence-based intervention is Community Aging in Place, Advancing Better Living for Elders (CAPABLE). [6-9] CAPABLE is a person-centered, delivered at home by clinicians (occupational therapists [OT] and registered nurses [RN]), 16-week intervention supported by assistive devices and home modification to improve function and factors that impact function (e.g., balance, pain, depression). It addresses modifiable intrinsic and extrinsic risk factors; and considers the psychological, environmental, and physical factors to enhance function of disabled adults and promotes aging-in-place. The intervention was adapted to fit the Michigan Medicaid home and community-based waiver program. [10] Despite availability of the adapted intervention, implementation of the intervention was limited.

A long-standing question in health care is how to implement evidence-based interventions in practice. On average, it takes 17 years for new knowledge generated from randomized controlled efficacy or effectiveness trials to be incorporated into practice. [11] To close the evidence-into-practice gap, a need exists to develop, evaluate, and implement solutions that are more effective at driving evidence into practice. Implementation strategies are key to explaining how to implement evidence-based interventions into practice. [12] Evidence on particular strategies is beginning to emerge. [13] Yet, limitations exist in regard to the packaging and testing of multi-modal strategies, specificity of strategies for replication, [14] and use across various types of settings. Usage of multi-modal implementation approaches are known to support both incremental single strategy effects, as well as a synergistic effect from all the strategies combined. [15] In addition, understanding how implementation strategies impact adoption and sustainability are topics of import to the field of implementation science. [16-20] Practice change and improved individual outcomes may occur with the use of proven implementation strategies that lead to adoption and sustainability of an intervention. [12]

We conducted a hybrid type III cluster-randomized controlled trial that compared implementation of the adapted intervention using a bundle of strategies to either internal facilitation (IF) or both IF and external facilitation (EF) plus usual care. The implementation strategy bundle included relationship, coalition, and team building; readiness to implement, leadership, and clinician attitude toward evidence assessments; intervention and facilitation training; interdisciplinary coordination; IF; EF; and audit and feedback. The 
adapted intervention addressed psychological, environmental, and physical factors to enhance function of disabled adults. [10]

The goal was to implement the intervention in the waiver to improve the ability of disabled or older adults (beneficiaries) to perform daily tasks. The study had three main objectives: (1) to evaluate adoption and sustainability of the intervention for two implementation strategies, IF and IF+EF; (2) to test clinician's self-efficacy and attitude toward evidence-based practice and deployment of an implementation strategy bundle on adoption and sustainability of the intervention; and (3) to examine the effect of the intervention on beneficiary outcomes. We hypothesized use in internal and external facilitation would increase adoption, sustainability, and improve beneficiary outcomes compared to internal facilitation alone. Beneficiary outcomes included activities of daily living (ADLs), instrumental activities of daily living (IADLs), pain, depression, falls, emergency department (ED) visits, and hospitalizations.

\section{Methods}

Study protocol is published elsewhere. [21] A brief description follows.

\section{Study design}

The design was a 2-arm, 3-year pragmatic hybrid type III [22-23] mixed method randomized controlled trial conducted at 18 waiver sites in the state of Michigan. [24-26] A hybrid design was chosen as it examines the effects of implementation strategies and intervention effectiveness for beneficiary outcomes. [23] The Knowledge-to-Action [27] model underpinned examination of outcomes, and the Consolidated Framework for Implementation Research [28-29] guided implementation. Data from clinicians were collected 9 months after training. Beneficiary data for the same period of time were extracted from the electronic health records (EHR), which included the last assessment prior to the clinician training (10/1/2019) and all assessments following implementation (10/2/2019 to 6/30/2020).

\section{Study setting and participants}

The settings were 18 Medicaid home and community-based waiver sites in Michigan that used the EHR system. Two Michigan sites using a different EHR were excluded. The waiver supports low-income at or below $300 \%$ of the Federal Poverty Level, nursing home eligible, disabled and older adults in the community to avoid institutionalization. Sites care for 400 to 2,500 beneficiaries and have 10 to 125 clinicians. Sites were contracted and clinicians were recruited via email at each site. Beneficiaries were recruited during usual care by clinicians, using a pocket aid to examine beneficiary needs in regard to the intervention. Beneficiaries could opt out and continue to receive care provided by the site, but their data were not extracted from the EHR or analyzed.

\section{Randomization and blinding}

To assure similarity of two trial arms, sites were paired in blocks using quality assessment scores (201517) and number of beneficiaries. A coin was flipped to determine arm assignment for each pair. Clinicians and beneficiaries were blinded to arm assignment. 


\section{Power analysis}

Given 18 sites ( 9 in each arm) available in Michigan, in the comparison of site-level outcome of adoption and sustainability, the detectable effect size with power of 0.80 in two-sided tests at .05 level of significance was Cohen's $d=1.41$. Given the sample size of clinicians of 539 , the average cluster size was approximately 45 , and with an assumed intra-class correlation coefficient (ICC) of 0.01 , the design effect factor was 1.45 , and the detectable effect size was Cohen's $d=0.29$. Given the sample size of 7,030 beneficiaries, the average cluster size was approximately 390 , and with an assumed intra-class correlation coefficient of 0.01 , the design effect factor was 4.9 , and the detectable effect size was Cohen's $\mathrm{d}=0.15$ for power of 0.80 in two-sided tests at .05 level of significance. These effect sizes are below $d=0.33-0.5$, the thresholds commonly used for clinical significance, therefore the study was powered to detect any meaningful differences between arms on clinician and beneficiary outcomes. [30, 31]

\section{Usual care}

Usual 1915(c) home and community-based services waiver care includes annual assessments, case management, and supports coordination by RNs and social workers (SWs) via home visits and phone calls. [32] Nineteen services are provided as needed and include adult day care, chore services (e.g., cleaning, laundry), community health worker, community transportation (e.g., to doctor's appointment), counseling, environmental modifications, and a fiscal intermediary. In addition, goods and services, home delivered meals, nursing services (e.g., medication management), personal emergency response system, private duty nursing/respiratory care, specialized medical equipment and supplies, training, personal care, medication management, lawn care, snow removal, cleaning, grocery shopping, and laundry are provided as needed.

\section{Evidence-based Intervention}

The 16-week intervention was implemented by OTs who conduct 6 home visits and provide assistive devices, RNs who conduct 4 home visits, and a handyman who provides home alterations such as installing devices and home modifications. [6-9] The team consults with the individuals to identify daily activity goals (e.g., taking a shower and walking to the bathroom) and evaluate barriers to achieving the goals to attain outcomes. OTs assist individuals to carry out ADLs and IADLs that are challenging, such as meal preparation, bathing, and dressing. RNs target pain, mood, fall prevention, incontinence management, and medication management. The intervention was adapted to include SWs to address social and emotional needs. [10]

\section{Implementation strategies}

Our bundle included 9 implementation strategies. First, informal relationships were built with sites which included monthly virtual meetings; and formal relationships were by memorandum of understanding, delineating the role and duties of IFs in each site. IFs were identified by each site's management team and approved by the Principal Investigator (PI). The EF was selected by the $\mathrm{PI}$ and was an OT who was an intervener at one of the sites in the pilot project. Second, readiness to implement and leadership were 
examined. Third, clinician attitude and self-efficacy were examined over time. Fourth, clinicians were trained in the intervention and IFs and the EF were trained in facilitation. IF training included an online 9module program which included an overview and the implementation plan and topics on problem solving, feedback, reflection, counseling, motivational interviewing, and remediation and a 60-minute synchronous session with the PI. EF training included completion of IF modules plus a 60-minute synchronous session with the PI which included a review of the IF role, challenges an IF may face, and how and when to facilitate with an IF. Fifth, a coalition of IFs met monthly to build capacity by sharing best practice use of implementation strategies. Sixth, IFs prompted interdisciplinary coordination among RNs, SWs, and OTs to promote teamwork, brainstorming, and problem solving to support beneficiary goal attainment. Seventh, IFs facilitated clinician implementation at all sites and the EF facilitated IFs at 9 sites (Arm 2). Eighth, an audit of implementation strategy and intervention fidelity occurred with feedback to IFs who worked with clinicians. Finally, the EF worked with IFs in Arm 2, providing feedback weekly. See the published protocol paper for detailed information and tools. [21]

\section{Measures}

Site, clinician, and beneficiary level data were collected using the Stages of Implementation (SIC) [33, 34] (sites); Organizational Readiness for Change (TCU-ORC), [35]; Evidence-Based Practice Attitude Scale (EBPAS), [36], General Self-efficacy (GSE), [37] (clinicians); and Minimum Data Set-Home Care (MDS-HC) [38] (beneficiaries) tools as described in detail in the protocol paper. [21] We also measured clinician and beneficiary characteristics and training completion.

\section{Outcomes}

Primary outcome was adoption and sustainability of the intervention measured via the SIC scores. Scoring of the SIC tool (range 0-100) is described in detail in the protocol paper. [21] Secondary outcomes were clinician attitudes toward evidence-based practice and self-efficacy and beneficiary ADLs (sum of 11 ADL items), IADLs (sum of 8 IADL items), pain (sum of 4 self-reported pain items), depression (sum of

3 self-reported mood items), and number of falls, ED visits, and hospitalizations. Intervention fidelity was not collected due to difficulty extracting data from the EHR.

\section{Study procedures}

Internal Review Board approvals were obtained, contracts (site, state, and EHR company) were executed, and IFs (each site) and the EF were selected and trained. Data (quality assessment scores, number of beneficiaries) were obtained from the state and sites were randomized. Clinicians were recruited, consented, and baseline data (characteristics, EBPAS, GSE, TCU-ORC) were obtained. Clinicians were trained and the intervention was provided to beneficiaries. We planned to collect the SIC (phone surveys) data monthly for 12 months, however, due to COVID-19, data were not collected in April through August 2020 , with the exception of 3 surveys from some of the sites. Clinician EBPAS and GSE were collected (survey) at baseline and 9 months. Beneficiary data prior to and after the intervention were obtained.

\section{Data analysis}


Stages of implementation scores were compared between trial arms using t-tests, and effect sizes (Cohen's d) were estimated as differences between means expressed in standard deviation units. The cutoffs for the interpretation of Cohen's d are 0.2 (small), 0.5 (medium), 0.8 (large) [39]. Characteristics of clinicians and beneficiaries were summarized by trial arm at baseline. Because of the turnover of clinicians at each site, constrained longitudinal model, with measures at baseline and at 9 months and a constraint of equality of means at baseline due to randomization, was used for the analysis of clinician data. With this analytical technique, data from all clinicians who completed baseline only, 9 months only, or both surveys were used. Random effects were used to account for nesting of clinicians within sites. For all beneficiaries, baseline data were available, and characteristics of beneficiaries without postintervention data were compared by trial arm to evaluate potential bias due to missing values. Postintervention data were analyzed in relation to trial arm with the adjustment for baseline version of the outcome, age, sex, and any baseline factors that differed in terms of missing values.

\section{Results}

\section{Site adoption and sustainability (Objective 1)}

All waiver sites ( $N=18 ; 100 \%)$ remained engaged in the trial during the entire study, a mean of 418.3 days (SD 11.54; range 496-224). The mean SIC scores (range 0-100) were 72.22 (standard deviation $[S D]=16.98)$ in the IF arm, and $61.33(S D=19.29)$ in the IF+EF arm. The difference was not statistically significant $(p=.22)$ with the sample size of 9 per arm, but corresponded to the medium clinically important effect size Cohen's $d=0.60$.

\section{Clinician implementation outcomes (Objective 2)}

There were 539 clinicians ( $\mathrm{n}=282 \mathrm{IF}$ arm, n=257 IF+EF arm) that comprised the study sample at baseline (see Figure 1). As shown in Tables 1 and 2, clinicians had similar socio-demographic characteristics and outcome values at baseline in two trial arms. All 539 (100\%) of the clinicians completed baseline and knowledge uptake surveys at baseline. Two hundred and sixty-four clinicians completed the 9-month survey ( $n=168$ in the IF arm 1; $n=96$ in the IF+EF arm). A total of 312 clinicians ( $n=133$ in the IF arm, $n=179$ in the IF+EF arm) had baseline but not 9 months measure, whereas 37 clinicians ( $n=19$ in the IF arm, $n=18$ in the IF+EF arm) had 9 months but not baseline data. The sample ICCs at baseline were below the value of .01 planned in a priori power analysis and were equal to .005 for self-efficacy (GSE) and .0007 for attitudes (EPBAS). There were no differences in clinician outcomes at month 9 (Table 4).

\section{Beneficiary outcomes (Objective 3 )}

The sample of beneficiaries 7,030 beneficiaries $(n=3,497$ arm $1 ; n=3,533$ arm 2) represented 9,752 beneficiaries served by the participating sites as of May 15, 2019. Of the beneficiaries served, 7,676 had the assessment near baseline of the trial (before October 1, 2019), 646 opted out ( $n=340 \mathrm{IF}$ arm; $n=306$ IF+EF arm, Figure 1), and the characteristics of 7,030 are in Table 2. Of these 7,030 beneficiaries, 384 in the IF arm and 291 did not have the second (post-intervention) MDH-HC assessment. The sample ICC's ranged from .01 (pain) to .039 (IADL) across beneficiary outcomes. The only significant difference by trial 
arm among beneficiaries with no post-intervention assessment was on age $(\mathrm{p}=.04$, Supplemental Table 1). Despite cluster randomization, IF+EF arm had a sizably larger proportion of African American participants (33\% versus $15 \%$ in the IF arm, Table 2 ), lower rate of recent falls ( $13 \%$ versus $22 \%$, Table 3 ), and lower pain intensity at baseline (Table 3). Per CONSORT guidelines, significance testing at baseline was not performed, but baseline recent falls and pain intensity were controlled for along with age and sex in the linear mixed effects models for post-intervention outcomes. Among the post-intervention outcomes, only depression was significantly lower in the IF+EF group compared to IF $(p=.04$, Table 5). No harms or unintended negative events attributed to the intervention were reported by beneficiaries.

\section{Discussion}

This trial sets an ambitious agenda to explore ways to adopt and sustain an evidence-based intervention across a Medicaid program. There is a significant amount of work that presents challenges and opportunities. First, implementation and evaluation with a hybrid III design in an under-resourced realworld setting force a delicate balance of study design and voluntary participation. Our work with sites in a standardized manner encourages local adaptation to optimize implementation. Similar to other studies, this trial was a success from a design perspective in that no sites dropped out. [40] Second, the selection of volunteer sites may limit heterogeneity in our sample. However, findings about stages of implementation will inform this intervention's adoption and sustainability and may be generalizable to other settings or populations. Findings from this trial are likely to generalize to other states and some community settings implementing the intervention because many challenges may be similar to a lowresource Medicaid setting of this trial. Third, this trial used 9 evidence-based implementation strategies, finding use of internal facilitation alone has less up-front cost and appears more sustainable. Like other implementation trials, multiple strategies were needed to achieve adoption and sustainability; [41, 42] and individuals involved in implementation performed formal and informal facilitation. [43] Fourth, the measurement approach involved significant effort in data collection from sites, clinicians, and beneficiaries with surveys, interviews, and extraction of secondary administrative and clinical data across disparate clinician contexts. Despite the challenges of data collection and management, the findings generate a broader understanding in Medicaid settings and facilitate effective use of evidence in underresourced, complex environments. Finally, past trials of the efficacy of CAPABLE with respect to beneficiary outcomes had similar results across multiple trials in low-income community-dwelling older adults with disabilities. [6-10,44] We expected to learn a great deal about beneficiary outcomes to further support efficacy of CAPABLE, but were unable to collect intervention fidelity data due to difficulty extracting data from the EHR.

Similar to other studies, we believe that this methodologic approach of measurement utilizing the SIC, which is comprised of the three dimensions of quality, quantity, and timing, is appropriate for a wide range of implementation and translational studies. [40] We note that the Michigan Department of Health and Human Services Long-term Care Division played a critical role in use of the intervention and implementation. Other states or settings may not have access to such an organization to support implementation. 


\section{Limitations}

The trial was limited by the number of sites in the State that utilized the same electronic health record (18 of 20). For this reason, only large effect sizes for site-level outcome of adoption and sustainability was detectable. To address this limitation, we also estimated the effect size to inform future dissemination and implementation trials. The ICCs for beneficiaries nested within sites were higher than planned based on the literature for outcomes other than pain. The nesting of beneficiaries within sites was accounted for analytically, but higher ICCs resulted in larger detectable effect sizes than planned in the a priori power analysis. Despite this limitation, observed point estimates of the differences between trial arms were small in magnitude, including the one significant difference between trial arms on beneficiary depression. Therefore, meaningful conclusions from this trial were not affected by somewhat higher than anticipated ICCs. Like other studies, we relied on self-reported data to measure clinician and beneficiary constructs $[45,46]$. We took several steps to mitigate this bias, including reminding the clinicians that their responses would not be shared with others and use of standardized data collection tools. In addition, the last few months of the trial may have been impacted by COVID, [47] yet the design and intervention implementation were sustained. Finally, even though all outcomes for clinicians and beneficiaries were specified a priori, one significant finding on beneficiary depression may be best explained by chance, supporting the overall conclusion of no differences between trial arms.

\section{Conclusions}

In what may be the first randomized controlled trial examining implementation of the evidence-based CAPABLE intervention, the level of facilitation did not enhance the capacity for adoption and sustainability in an under-resourced Medicaid setting that cares for disabled and older adults. This suggests that only internal facilitation may be warranted. These findings further support the merits of less intensive implementation approaches that ensure adequate training and ongoing facilitation to support clinicians attempting to implement a new intervention. Future studies should evaluate factors that predict optimal implementation, particularly, internal facilitation.

\section{Abbreviations}

Activities of daily living (ADL); Electronic health record (EHR); Emergency Department (ED); EvidenceBased Practice Attitude Scale (EBPAS); External facilitation/facilitator (EF); General Self-Efficacy (GSE); Instrumental activities of daily living (IADL); Internal facilitation/facilitator (IF); Minimum Data Set-Home Care (MDS-HC); Occupational Therapist (OT); Organizational Readiness for Change (TCU-ORC); Principal Investigator (PI); Registered Nurse (RN); Social Worker (SW); Stages of Implementation Completion (SIC)

\section{Declarations}


Ethics approval and consent to participate: Protocol described here was approved by the University 19061-H Institutional Review Board. Participants were informed about aims of study and methods for protection of data privacy. Informed consent was obtained from clinicians and implied by beneficiaries who do not opt out. Reports will contain aggregate data allowing no identification of individual participants.

Consent for publication: Not applicable.

Availability of data and material: The datasets generated during and/or analyzed will be available upon request from the investigators.

Competing interests: The authors declare they have no competing interests.

Funding: The study is funded by National Institutes of Aging 1 R15 AG058193-01A1 (9/1/2018 to 8/30/2021).

Authors' contributions: SS and MS (study concept and design, selection of methods, acquisition of sites and subjects and/or data, analysis plan, and preparation of manuscript); TB (data cleaning, analysis, preparation of tables, figures, and manuscript); AS (study concept and design, analysis plan, and preparation of manuscript).

Acknowledgements: We sincerely thank the clinicians at the 18 Medicaid sites for their time and effort.

\section{References}

1. Fenelon A, Mawhorter S. Housing affordability and security issues facing older adults in the United States. Public Policy Aging Rep. 2020;31:30-2.

2. Cornman JC, Glei DA, Weinstein M. Change in mobility: consistency of estimates and predictors across studies of older adults. J Geront B Psychol Sci Soc Sci. 2021;76:209-18.

3. Kamil-Rosenberg S, Greaney ML, Garber CE. Health-related and sociodemographic correlates of meeting the muscle strengthening exercise recommendations in middle-aged and older adults with and without disabilities. Sport Sci Health. 2021;17:201-11.

4. Chyr LC, Drabo EF, Fabius CD. Patterns and predictors of transitions across residential care settings and nursing homes among community-dwelling older adults in the United States. Gerontologist. 2020;60:1495-503.

5. McGrath R, Stastny S, Casperson S, Jahns L, Roemmich J, Hackney KJ. Daily protein intake and distribution of daily protein consumed decreases odds for functional disability in older Americans. J Aging Health. 2020;32:1075-83.

6. Szanton SL, Wolff JW, Leff B, Thorpe RJ, Tanner EK, Boyd C, et al. CAPABLE trial: a randomized controlled trial of nurse, occupational therapist and handyman to reduce disability among older adults: rationale and design. Contemp Clin Trials. 2014;38:102-12. 
7. Szanton SL, Thorpe RJ, Boyd C, Tanner EK, Leff B, Agree E, et al. Community aging in place, advancing better living for elders (CAPABLE): a bio-behavioral-environmental intervention to improve function and health-related quality of life in disabled older adults. J Am Geriatr Soc. 2011;59:231420.

8. Szanton SL, Leff B, Wolff JL, Roberts L, Gitlin LN. Home-based care program reduces disability and promotes aging in place. Health Aff. 2016;35:1558-63.

9. Szanton SL, Xue Q, Leff B, Guralnik J, Wolff JL, Tanner EK, et al. Effect of a biobehavioral environmental approach on disability among low-income older adults: a randomized clinical trial. JAMA Intern Med. 2019;179:204-11.

10. Spoelstra S, Sikorskii A, Gitlin L, Schueller M, Kline M, Szanton SL. Dissemination of CAPABLE model of care in a Medicaid waiver program to improve physical function. J Am Geriatr Soc. 2018;67:36370.

11. Institute of Medicine (US) Committee on Quality of Health Care in America. Crossing the quality chasm: a new health system for the 21 st century. Washington (DC): National Academies Press (US); 2001. http://www.ncbi.nlm.nih.gov/books/NBK222274/. From.

12. Stange KC, Breslau ES, Dietrich AJ, Glasgow RE. State-of-the-art and future directions in multilevel interventions across the cancer control continuum. J Natl Cancer Inst Monogr. 2012;2012:20-31.

13. Grimshaw JM, Eccles MP, Lavis JN, Hill SJ, Squires JE. Knowledge translation of research findings. Implement Sci. 2012;7:50.

14. Proctor EK, Powell BJ, McMillen JC. Implementation strategies: recommendations for specifying and reporting. Implement Sci. 2013;8:139.

15. Stirman SW, Kimberly J, Cook N, Calloway A, Castro F, Charns M. The sustainability of new programs and innovations: a review of the empirical literature and recommendations for future research. Implement Sci. 2012;7:17.

16. Scheirer MA, Dearing JW. An agenda for research on the sustainability of public health programs. Am J Public Health. 2011;101:2059-67.

17. Milat AJ, King L, Newson R, Wolfenden L, Rissel C, Bauman A, et al. Increasing the scale and adoption of population health interventions: experiences and perspectives of policy makers, practitioners, and researchers. Health Res Policy Syst. 2014;12:18.

18. Chambers DA. Advancing sustainability research: challenging existing paradigms. J Public Health Dent. 2011;71(Suppl 1):99-100.

19. Tricco AC, Ashoor HM, Cardoso R, MacDonald H, Cogo E, Kastner M, et al. Sustainability of knowledge translation interventions in healthcare decision-making: a scoping review. Implement Sci. 2015;11:55.

20. Willis CD, Riley BL, Stockton L, Abramowicz A, Zummach D, Wong G, et al. Scaling up complex interventions: insights from a realist synthesis. Health Res Policy Sys. 2016;14:88.

21. Spoelstra SL, Schueller M, Sikorskii A. Testing an implementation strategy bundle on adoption and sustainability of evidence to optimize physical function in community-dwelling disabled and older 
adults in a Medicaid waiver: a multi-site pragmatic hybrid type III protocol. Implement Sci. 2019;14:60.

22. Meffert SM, Neylan TC, Chamber DA, Verdeli H. Novel implementation research designs for scaling up global mental health care: overcoming translational challenges to address the world's leading cause of disability. Int J Ment Health Syst. 2016;10:19.

23. Curran GM, Bauer M, Mittman B, Pyne JM, Stetler C. Effectiveness-implementation hybrid designs: combining elements of clinical effectiveness and implementation research to enhance public health impact. Med Care. 2012;50:217-26.

24. Palinkas LA, Aarons GA, Horwitz S, Chamberlain P, Hurlburt M, Landsverk J. Mixed method designs in implementation research. Adm Policy Ment Health. 2011;38:44-53.

25. Green CA, Duan N, Gibbons RD, Hoagwood KE, Palinkas LA, Wisdom JP. Approaches to mixed methods dissemination and implementation research: methods, strengths, caveats, and opportunities. Adm Policy Ment Health. 2015;42:508-23.

26. Palinkas LA, Horwitz SM, Green CA, Wisdom JP, Duan N, Hoagwood K. Purposeful sampling for qualitative data collection and analysis in mixed method implementation research. Adm Policy Ment Health. 2015;42:533-44.

27. Field B, Booth A, llott I, Gerrish K. Using the Knowledge to Action Framework in practice: a citation analysis and systematic review. Implement Sci. 2014;9:172.

28. Damschroder LJ, Aron DC, Keith RE, Kirsh SR, Alexander JA, Lowery JC. Fostering implementation of health services research findings into practice: a consolidated framework for advancing implementation science. Implement Sci. 2009;4:50.

29. Breimaier HE, Heckemann B, Halfens RJ, Lohrmann C. The Consolidated Framework for Implementation Research (CFIR): a useful theoretical framework for guiding and evaluating a guideline implementation process in a hospital-based nursing practice. BMC Nursing. 2015;14:43.

30. Sloan JA, Cella D, Hays RD. Clinical significance of patient-reported questionnaire data: another step toward consensus. J Clin Epidemiol. 2005;58:1217-9.

31. Norman GR, Sloan JA, Wyrwich KW. Interpretation of changes in health-related quality of life: the remarkable universality of half a standard deviation. Med Care. 2003;41:582-92.

32. Michigan Department of Health \& Human Services. 1951c Home and Community-Based Services. https://www.michigan.gov/mdhhs (2021). Accessed 18 May 2021.

33. Saldana L. The stages of implementation completion for evidence-based practice: protocol for a mixed methods study. Implement Sci. 2014;9:43.

34. Chamberlain P, Brown $\mathrm{CH}$, Saldana L. Observational measure of implementation progress in community based settings: the Stages of implementation completion (SIC). Implement Sci. 2011;6:116.

35. Gagnon MP, Attieh R, Ghandour EK, Légaré F, Ouimet M, Estabrooks CA, et al. A systematic review of instruments to assess organizational readiness for knowledge translation in health care. PLoS One. 2014;9:e114338. 
36. Aarons GA. Mental health provider attitudes toward adoption of evidence-based practice: the Evidence-Based Practice Attitude Scale (EBPAS). Ment Health Serv Res. 2004;6:61-74.

37. Schwarzer R, Jerusalem M. Generalized Self-Efficacy scale. In: Weinman J, Wright S, Johnston M, editors. Measures in health psychology: a user's portfolio. Causal and control beliefs. Windsor: NFERNELSON; 1995. pp. 35-7.

38. Fries BE, Shugarman LR, Morris JN, Simon SE, James M. A screening system for Michigan's homeand community-based long-term care programs. Gerontologist. 2002;42:462-74.

39. Cohen J. Statistical power analysis for the behavioral sciences. 2nd ed. Hillsdale: Lawrence Erlbaum Associates; 1988.

40. Brown $\mathrm{CH}$, Chamberlain P, Saldana L, Padgett C, Wang W, Cruden G. Evaluation of two implementation strategies in 51 child county public service systems in two states: results of a cluster randomized head-to-head implementation trial. Implement Sci. 2014;9:134.

41. Reynolds SS, Woltz P, Keating E, Neff J, Elliott J, Hatch D, et al. Results of the CHlorhexidine Gluconate Bathing implementation intervention to improve evidence-based nursing practices for prevention of central line associated bloodstream infections Study (CHanGing BathS): a stepped wedge cluster randomized trial. Implement Sci. 2021;16:45.

42. Wagner GJ, Wanyenze RK, Beyeza-Kashesya J, Gwokyalya V, Hurley E, Mindry D, et al. "Our Choice" improves use of safer conception methods among HIV serodiscordant couples in Uganda: a cluster randomized controlled trial evaluating two implementation approaches. Implementat Sci. 2021;16:41.

43. Dogherty EJ, Harrison MB, Baker C, Graham ID. Following a natural experiment of guideline adaptation and early implementation: a mixed-methods study of facilitation. Implement Sci. 2012;7:9.

44. Szanton SL, Leff B, Li Q, Breysse J, Spoelstra S, Kell J, et al. CAPABLE program improves disability in multiple randomized trials. J Am Geriatr Soc. 2021;69:3631-40.

45. Garnett A, Ploeg J, Markle-Reid M, Strachan PH. Self-report tools for assessing physical activity in community-living older adults with multiple chronic conditions: a systematic review of psychometric properties and feasibility. Can J Aging. 2020;39:12-30.

46. Hogue A, Porter N, Bobek M, MacLean A, Bruynest L, Jensen-Doss A, et al. Online training of community therapists in observational coding of family therapy techniques: reliability and accuracy. Adm Policy Ment Health. 2022;49:139-51.

47. Harper L, Kalfa N, Beckers GMA, Kaefer M, Nieuwhof-Leppink AJ, Fossum M, et al. The impact of COVID-19 on research. J Pediatr Urol. 2020;16:715-6.

\section{Tables}

Table 1 Socio-demographic characteristics of the clinician sample $(n=539)$ 


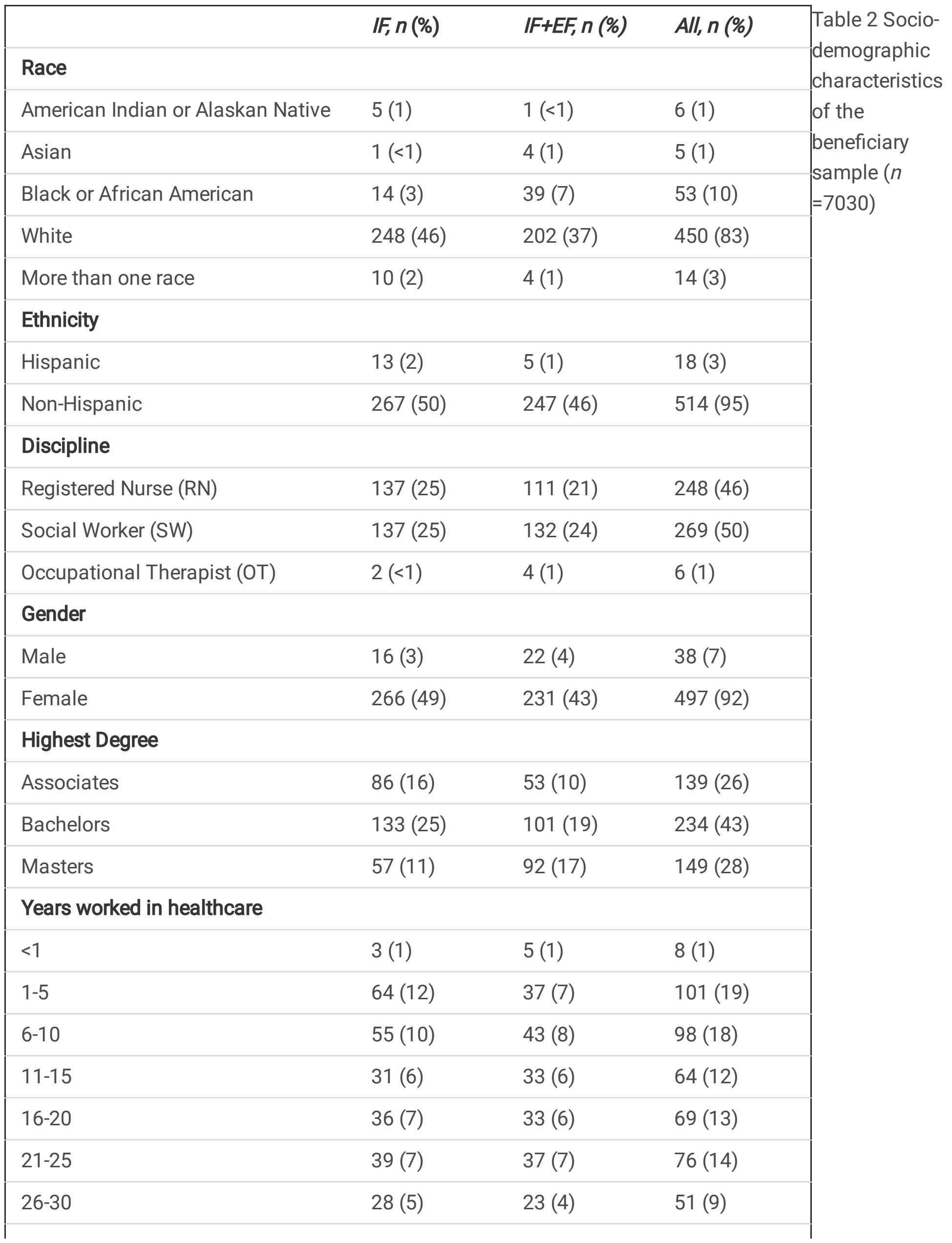




\begin{tabular}{|c|c|c|c|c|c|}
\hline$>30$ & \multicolumn{2}{|l|}{$25(5)$} & $45(8)$ & $70(13)$ & \\
\hline \multicolumn{5}{|l|}{ Years worked in waiver } & \\
\hline$<1$ & \multicolumn{2}{|l|}{$44(8)$} & $28(5)$ & $72(13)$ & \\
\hline $1-5$ & \multicolumn{2}{|c|}{$148(27)$} & $122(23)$ & $270(50)$ & \\
\hline $6-10$ & \multicolumn{2}{|l|}{$50(9)$} & $45(8)$ & $95(18)$ & \\
\hline $11-15$ & \multicolumn{2}{|l|}{$13(2)$} & $19(4)$ & $32(6)$ & \\
\hline $16-20$ & \multicolumn{2}{|l|}{$11(2)$} & $22(4)$ & $33(6)$ & \\
\hline $21-25$ & \multicolumn{2}{|l|}{$9(2)$} & $17(3)$ & $26(5)$ & \\
\hline $26-30$ & \multicolumn{2}{|l|}{$4(1)$} & $2(<1)$ & $6(1)$ & \\
\hline \multicolumn{5}{|c|}{ Mean (SD) } & \\
\hline \multirow[t]{2}{*}{ Age } & \multicolumn{2}{|c|}{43.07 (11.57) } & $47.00(11.59)$ & $44.92(11.74)$ & \\
\hline & \multicolumn{2}{|c|}{$I F, n(\%)$} & \multicolumn{2}{|c|}{$I F+E F, n(\%)$} & $l, n(\%)$ \\
\hline \multicolumn{6}{|l|}{ Race } \\
\hline $\begin{array}{l}\text { American Indian or Alaskan } \\
\text { Native }\end{array}$ & $25(<1)$ & \multicolumn{2}{|c|}{$9(<1)$} & \multicolumn{2}{|l|}{$34(1)$} \\
\hline Asian & $25(<1)$ & \multicolumn{2}{|c|}{$25(<1)$} & $50(1)$ & \\
\hline Black or African American & $541(8)$ & \multicolumn{2}{|c|}{$1228(17)$} & $1769(25)$ & \\
\hline Hawaiian or South Pacific & $3(<1)$ & \multicolumn{2}{|c|}{$4(<1)$} & $7(<1)$ & \\
\hline White & $2735(39)$ & \multicolumn{2}{|c|}{$2122(30)$} & $4857(69)$ & \\
\hline More than one race & $49(1)$ & \multicolumn{2}{|c|}{$33(1)$} & $82(1)$ & \\
\hline \multicolumn{6}{|l|}{ Ethnicity } \\
\hline Hispanic & $108(2)$ & \multicolumn{2}{|c|}{$97(1)$} & $205(3)$ & \\
\hline Non-Hispanic & $3357(48)$ & \multicolumn{2}{|c|}{3401 (48) } & $6758(96)$ & \\
\hline \multicolumn{6}{|l|}{ Gender } \\
\hline Male & $1137(16)$ & \multicolumn{2}{|c|}{$1130(16)$} & 2267 (32) & \\
\hline \multirow[t]{2}{*}{ Female } & $2360(34)$ & \multicolumn{2}{|c|}{$2403(34)$} & $4763(68)$ & \\
\hline & \multicolumn{3}{|l|}{ Mean (SD) } & & \\
\hline Age & $\begin{array}{l}69.50 \\
(14.58)\end{array}$ & & 9 (14.02) & 70.35 (14.32) & \\
\hline
\end{tabular}


Table 3 Baseline values of the outcomes and potential mediators by trial arm

\begin{tabular}{lll} 
Outcome or mediator & IF Mean (SD) & IF+EF Mean (SD) \\
\hline Clinician climate & $35.78(6.06)$ & $35.02(6.61)$ \\
\hline Clinician culture & $12.54(1.92)$ & $12.35(2.19)$ \\
\hline Clinician training & $8.18(1.61)$ & $8.12(1.56)$ \\
\hline Clinician motivation & $13.25(3.40)$ & $13.95(3.28)$ \\
\hline Clinician pressure to change & $10.49(2.30)$ & $9.70(2.48)$ \\
\hline Clinician leadership & $34.11(9.07)$ & $32.91(10.21)$ \\
\hline Clinician attitude & $105.90(18.27)$ & $106.25(19.10)$ \\
\hline Clinician self-efficacy & $32.20(3.80)$ & $32.19(3.84)$ \\
\hline Beneficiary ADL & $32.05(16.76)$ & $33.48(15.90)$ \\
\hline Beneficiary IADL & $37.95(10.04)$ & $37.28(9.41)$ \\
\hline Beneficiary pain & $2.56(3.08)$ & $3.01(3.20)$ \\
\hline Beneficiary depression & $1.44(1.99)$ & $1.05(1.79)$ \\
\hline & $\mathbf{n}(\%)$ & $\mathbf{n}(\%)$ \\
\hline Beneficiary falls & $177(22)$ & $113(13)$ \\
\hline Beneficiary ED visits & $450(13)$ & $421(12)$ \\
\hline Beneficiary hospitalizations & $495(14)$ & $450(13)$
\end{tabular}

Table 4 Post-intervention differences between trial arms for clinicians 


\begin{tabular}{lllll}
$\begin{array}{l}\text { Outcome or } \\
\text { mediator }\end{array}$ & $\begin{array}{l}\text { IF Mean } \\
\text { change (SE) }\end{array}$ & $\begin{array}{l}\text { IF+EF Mean } \\
\text { change }(S E)\end{array}$ & $\begin{array}{l}\text { Difference between } \\
\text { arms }(95 \% \text { CI) }\end{array}$ & $\begin{array}{l}\text { p-Value differences } \\
\text { between arms }\end{array}$ \\
\hline $\begin{array}{l}\text { Clinician self- } \\
\text { efficacy }\end{array}$ & $-2.34(0.21)$ & $-2.57(0.30)$ & $0.23(-0.51,0.97)$ & 0.54 \\
\hline $\begin{array}{l}\text { Clinician attitude - } \\
\text { fit }\end{array}$ & $*$ & $*$ & $*$ & $*$ \\
\hline $\begin{array}{l}\text { Clinician attitude - } \\
\text { limitations }\end{array}$ & $0.00(0.11)$ & $-0.06(0.11)$ & $0.06(-0.27,0.39)$ & 0.68 \\
\hline $\begin{array}{l}\text { Clinician attitude - } \\
\text { openness }\end{array}$ & $0.08(0.08)$ & $0.00(0.08)$ & $0.08(-0.13,0.29)$ & 0.45 \\
\hline $\begin{array}{l}\text { Clinician attitude - } \\
\text { monitoring }\end{array}$ & $0.01(0.14)$ & $0.18(0.14)$ & $-0.17(-0.66,0.32)$ & 0.44 \\
\hline $\begin{array}{l}\text { Clinician attitude - } \\
\text { requirements }\end{array}$ & $0.15(0.09)$ & $0.09(0.10)$ & $0.06(-0.22,0.35)$ & 0.64 \\
\hline $\begin{array}{l}\text { Clinician attitude - } \\
\text { employability }\end{array}$ & $-0.17(0.07)$ & $-0.04(0.11)$ & $-0.12(-0.36,0.11)$ & 0.29 \\
\hline $\begin{array}{l}\text { Clinician attitude - } \\
\text { feedback }\end{array}$ & $0.10(0.11)$ & $-0.01(0.12)$ & $0.11(-0.24,0.47)$ & 0.50 \\
\hline $\begin{array}{l}\text { Clinician attitude - } \\
\text { burden }\end{array}$ & $0.13(0.15)$ & $0.04(0.14)$ & $0.09(-0.42,0.59)$ & 0.69 \\
\hline $\begin{array}{l}\text { Clinician attitude - } \\
\text { appeal }\end{array}$ & $0.06(0.08)$ & $-0.11(0.08)$ & $0.17(-0.06,0.41)$ & 0.13 \\
\hline $\begin{array}{l}\text { Clinician attitude - } \\
\text { divergence }\end{array}$ & $0.07(0.09)$ & $0.14(0.09)$ & $-0.07(-0.31,0.17)$ & 0.57 \\
\hline $\begin{array}{l}\text { Clinician attitude - } \\
\text { balance }\end{array}$ & $0.11(0.11)$ & $0.21(0.10)$ & $-0.10(-0.47,0.27)$ & 0.54 \\
\hline $\begin{array}{l}\text { Clinician attitude - } \\
\text { total }\end{array}$ & -103.49 & $-103.52(0.92)$ & $0.03(-0.08,0.14)$ & 0.70 \\
\hline 0.92$)$ & & & \\
\hline
\end{tabular}

*Algorithm did not converge

Table 5 Post-intervention differences between trial arms for beneficiaries 


\begin{tabular}{lllll} 
Outcome & $\begin{array}{l}\text { IFLSMean } \\
(S E)\end{array}$ & $\begin{array}{l}\text { IF+EF LS } \\
\text { Mean (SE) }\end{array}$ & $\begin{array}{l}\text { Difference between } \\
\text { arms }(95 \% \text { CI) }\end{array}$ & $\begin{array}{l}\text { p-value for differences } \\
\text { between arms }\end{array}$ \\
\hline ADL & $\begin{array}{l}26.13 \\
(0.20)\end{array}$ & $25.85(0.19)$ & $(-0.16,0.73)$ & 0.73 \\
\hline IADL & $\begin{array}{l}29.54 \\
(0.17)\end{array}$ & $29.24(0.17)$ & $(-0.11,0.72)$ & 0.15 \\
\hline Pain & $7.52(0.13)$ & $7.44(0.13)$ & $(-0.23,0.39)$ & 0.61 \\
\hline Pain intensity & $2.71(0.08)$ & $2.70(0.08)$ & $(-0.18,0.20)$ & 0.92 \\
\hline Depression & $1.30(0.05)$ & $1.17(0.05)$ & $(0.01,0.24)$ & 0.04 \\
\hline & $\begin{array}{l}\text { Adjusted } \\
\text { rate (SE) }\end{array}$ & $\begin{array}{l}\text { Adjusted } \\
\text { rate (SE) }\end{array}$ & $\begin{array}{l}\text { OR }(95 \% \text { CI) for IF v. } \\
\text { IFF }(1 \text { v. } 2)\end{array}$ & p-value for differences \\
\hline Falls & $15.44 \%$ & $13.56 \%$ & $1.16(0.93,1.45)$ & 0.18 \\
\hline Recent falls & $5.40 \%$ & $4.62 \%$ & $1.18(0.71,1.95)$ & 0.52 \\
\hline ED visits & $0.92 \%$ & $0.90 \%$ & $0.98(0.79,1.22)$ & 0.86 \\
\hline Hospitalizations & $1.48 \%$ & $1.44 \%$ & $1.01(0.72,1.42)$ & 0.95
\end{tabular}

\section{Figures}

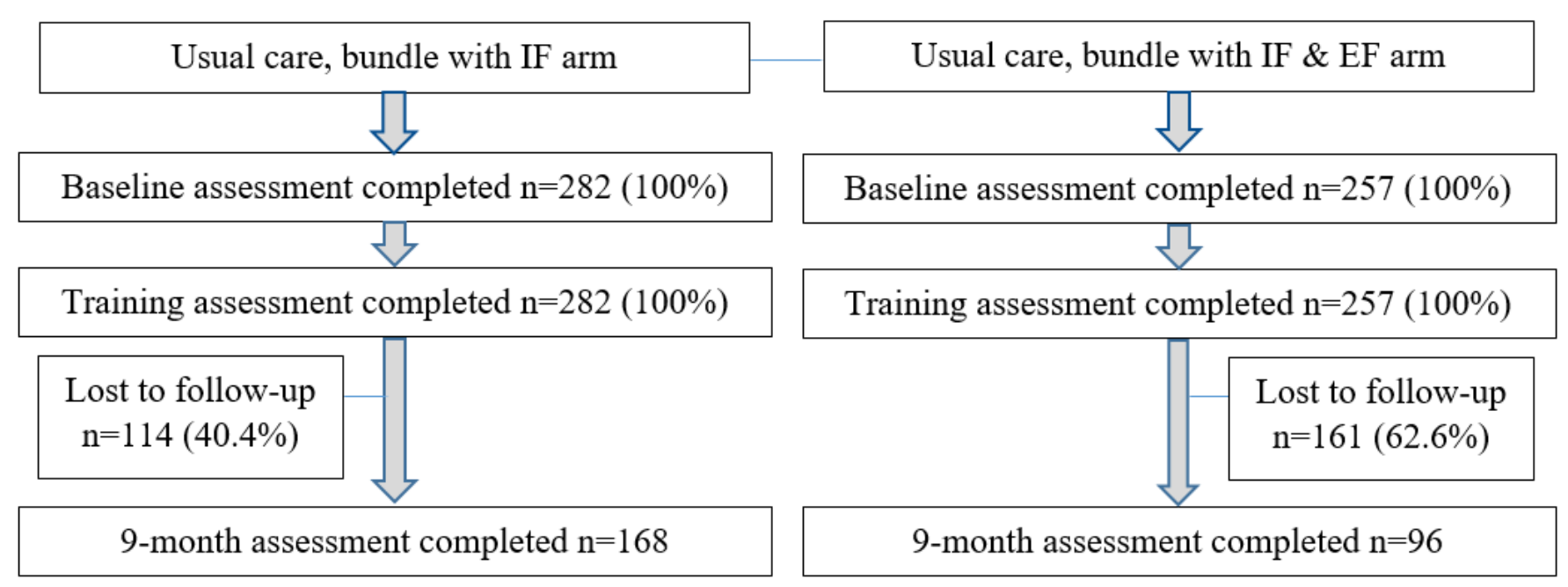

\section{Figure 1}

Consort diagram of clinician study participants 


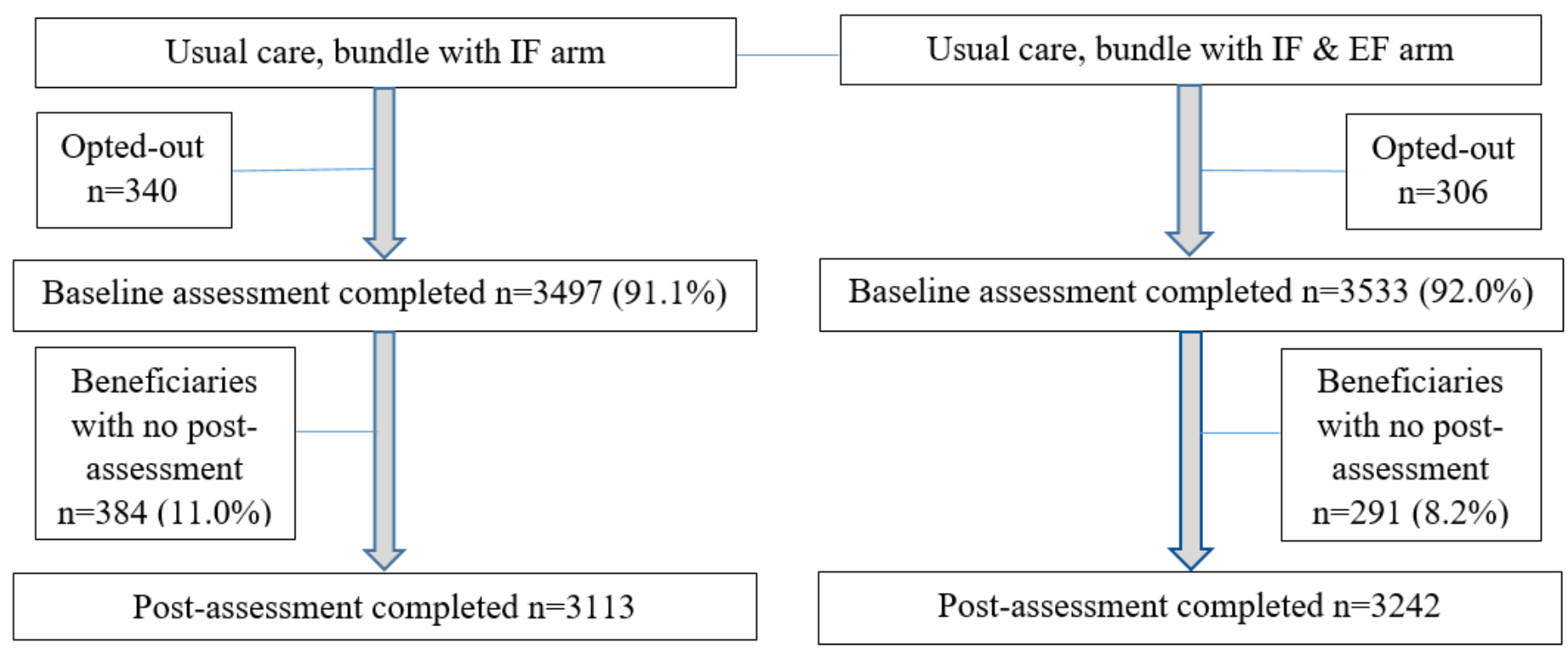

Figure 2

Consort diagram of beneficiary study participants

\section{Supplementary Files}

This is a list of supplementary files associated with this preprint. Click to download.

- SupplementaryMaterialTable1.docx

- CONSORT2010ChecklistSS.doc

- StaRIchecklistSS.docx 